\title{
A Quality Improvement Study Project to Improve Post Cesarean Section Surgical Site Infection Surveillance in a District Hospital in Kigali City
}

\author{
Evode Uwamungu ${ }^{1,3}$, William Rutagengwa ${ }^{2}$, Jenae Logan ${ }^{3}$, Pascal Nkubito ${ }^{2} \&$ Rex Wong $^{3}$ \\ ${ }^{1}$ Management Sciences for Health, Rwanda \\ ${ }^{2}$ Kacyiru Hospital, Rwanda \\ ${ }^{3}$ University of Global Health Equity, Rwanda \\ Correspondence: Evode Uwamungu, Management Sciences for Health, Rwanda.
}

Received: January 12, 2019

Accepted: March 7, 2019

Online Published: March 11, 2019

doi:10.5430/jms.v10n2p18

URL: https://doi.org/10.5430/jms.v10n2p18

\begin{abstract}
Post-caesarean surgical site infection (PCSI) is one of the most common cesarean section-related complications. In low- and middle-income countries (LMIC), PCSI prevalence is often under-reported and inaccurate because LMIC surveillance systems are often unable to detect PCSIs developed after discharge; this can ultimately wrongly inform the decision-making related to reducing PCSIs.

This paper describes the establishment of a post-discharge PCSI surveillance system for identification of PCSI rate in a district hospital in Rwanda.

A total of 540 women underwent CS in the hospital from November 2017 to February 2018, and 536 (99.3\%) consented to participate in the surveillance. Among those consented, 22 had no telephone and 174 could not be reached by telephone despite multiple attempts. At the end of this study, a total of 340 women completed the entire surveillance period. The total PCSI rate was $11.5 \%$.Out of all PCSIs, $21 \%$ were detected during hospitalization period and $79 \%$ were detected during the post-discharge period.

The PCSI surveillance system developed in this project covered the 30-day period after surgery and provided a more accurate estimate of PCSI rate. The system was able to track PCSIs developed after a patient was discharged from the hospital. Long term sustainability of the project must be evaluated.
\end{abstract}

Keywords: post cesarean section infections, surveillance, risk factors, cesarean section

\section{Introduction}

Cesarean section (CS) is a surgical procedure that assists in the delivery of a baby and is the most performed surgery worldwide (Soto-Vega et al., 2015; Yadav, Kaur, Yadav, \& Thakur, 2016). On average, in Sub-Saharan Africa, about $8 \%$ of deliveries in public health facilities and $12 \%$ in private facilities are CS (Yaya, Uthman, Amouzou, \& Bishwajit, 2018). CS can save lives of both mothers and infants, but it is not without negative consequences (Soto-Vega et al., 2015; Yadav, Kaur, Yadav, \& Thakur, 2016). There are many possible complications of CS, including bleeding, urogenital injuries, and deep venous thrombosis. Post-caesarean surgical site infection (PCSI) is one of the most common CS related complications (Yadav, Kaur, Yadav, \& Thakur, 2016).

Women with CS have an estimated 5- to 20-fold increase in incidence of infection compared with women delivering without CS (Conroy et al., 2012; Bagnall et al., 2009; Maruta, 2015); which in turn can cause unnecessary maternal morbidity and mortality, increased financial and emotional stress for families, and higher expenditure by the health care facilities (Lukas et al., 2016; Kelly \& Monson, 2012; Conroy et al., 2012; Bagnall, Vig \& Trivedi, 2009).

A properly functioning Hospital Acquired Infections (HAI) surveillance and control system can help health care providers to reduce about $10 \%$ to $35 \%$ of surgical site infections (Haley et al., 1985). In low- and middle-income countries (LMIC), due to the challenges in establishing proper reporting systems, PCSI prevalence is usually considered under-estimated and inaccurate (Allegranzi \& Pittet, 2008; Lukas et al., 2016). The reported PCSI rates in LMIC varies substantially from $0.2 \%$ to $48 \%$ due to a number of factors including inadequate surveillance systems that often underestimate the true incidence of PCSI (Bizimana et al., 2016; Gur et al., 2015; Nguhuni et al., 2017; Srun et al., 2013). 
Since surgical site infection can develop within 30 days of an operative procedure, it is estimated that $27 \%$ to $95 \%$ of PCSI develop after women are discharged from hospitals; nevertheless, most hospitals only report PCSI that are recorded during hospitalization (Bizimana et al., 2016; Chu, Maine, \& Trelles, 2015; Eggimann \& Pittet, 2001; Humphreys, 2009; Mpogoro et al., 2014; Owens \& Stoessel, 2008). The lack of accurate and consistent data could wrongly inform decision-making regarding the reduction of PCSIs. There is an increasing need to establish post-discharge surveillance systems to obtain accurate rates of PCSI (Mitt et al., 2002; Petherick et al., 2006; Humphreys, 2009; Dhar et al., 2014; Gould, 2007). Among the various post-discharge surveillance methods, which include direct observation of the wound by health professionals, questionnaires, and clinical document auditing, telephone surveillance is one of the most feasible and cost effective (Petherick et al., 2006; Taylor et al., 2003; Nguhuni et al., 2017).

In Rwanda, most HAI surveillance consists only of recording infections that occur during hospital stays; any infection that is developed after discharge is undetected. The real magnitude of PCSI may therefore be grossly under reported. Without such accurate information, organizational planning and allocation of resources may be misinformed. More importantly than the accuracy of data is that most patients do not develop infections until after being discharged from the hospitals, and they may not know to seek timely care. This has the potential to lead to negative health outcomes. There is a need to establish a surveillance system that follows up with patients to capture and report the actual PCSI rate. This paper describes the establishment of a post-discharge PCSI surveillance system in a district hospital in Rwanda.

\section{Methods}

\subsection{Setting}

This study was conducted in an 86-bed district hospital located in Kigali City, Rwanda. The hospital provides maternal and child health services and performs about five to eight cesarean sections per day. The hospital reported that the average PCSI rate was $1.6 \%$ in 2016.

\subsection{Study Design and Sample}

This project set up a new surveillance system to determine the PCSI rate inclusive of infections developed during the post-hospitalization period. All women who had cesarean section deliveries in the hospital during the period of November 2017 to February 2018 and consented to participate were included in the surveillance. Any women who died during the surgical operation were excluded.

\subsection{Establishing the Surveillance System}

\subsubsection{Overview}

A project team was established to develop the new PCSI surveillance system. The surveillance system was established to collect data during hospitalization as well as up to 30 days after patient was discharged. A surveillance team was formed to develop and implement a data collection form. The surveillance results were reported to the hospital management team for future PCSI-related planning.

\subsubsection{The Project Team}

The project team was formed in October 2017. The team included health care professionals from different disciplines, including physicians and nurses working in the maternity unit, the infection prevention and control director, and other health system professionals. The project team conducted extensive research on the available and acceptable PCSI surveillance methods.

\subsubsection{The Data Collection Tool}

The center-piece of the PCSI surveillance system was the development of a simple and easy to use data collection tool to gather an accurate estimate of PCSIs. Considering the availability of laboratory services during hospitalization and the feasibility of having patients to seek laboratory services post-discharge, the data collection tool the project team decided to use required minimal laboratory support and was thus more realistic for use in resource-limited settings. The data collection tools were adapted from the Saskatchewan Infection Prevention and Control Surveillance Program Protocol (Saskatchewan Ministry of Health, 2015) and the CDC National Nosocomial Infections Surveillance guidelines. These tools captured PCSI during hospitalization and post-discharge. The data collection tools were pre-tested by clinical staff.

The "In-Patient and Readmissions PCSI Surveillance Form" (appendix 1) was designed to capture the PCSI developed during inpatient stay after delivery as well as PCSI in women who were readmitted to the hospital within 30 days after the previous discharge. The form collected information on patient background and demographics, 
details on the operation procedure (date of operation, existence of a ruptured membrane before procedure and the time period in which it happened, a preoperative condition following the American Society of Anesthesiologists (ASA) scoring system, emergency nature of the operation, wound classification, interruption of skin closure, type of skin closure, blood loss or postpartum hemorrhage and prescription of prophylaxis antibiotic), and presence of PCSI.

\subsubsection{Surveillance Team}

The data collection team included four nurses who were working in the maternity unit at the hospital; one of them was also the hospital's Infection Prevention and Control Program supervisor. The four data collectors were provided with data collection guidelines and training on the data collection process.

\subsubsection{Surveillance Method}

\subsubsection{PCSI During Hospitalization}

The data collectors obtained information for this form through: 1) direct observation, 2) interview with patients, and 3) review of patient records.

The completed forms were kept in the patients' medical files until discharge. When the patient was discharged, the data collectors would keep the forms in a locked cupboard in their office for post-discharge follow-up.

\subsubsection{Post Discharge Period}

The "Post-discharge Telephone Surveillance Form" (appendix 2) was used to collect patient information related to PCSI on the $10^{\text {th }}$ and $30^{\text {th }}$ day after CS if a woman was discharged from the hospital before the $10^{\text {th }}$ day post-CS.

Data collectors followed up with patients via telephone on the $10^{\text {th }}$ and $30^{\text {th }}$ day post CS between 8:00AM and 6:00PM to minimize disruption to patients' lives. The $10^{\text {th }}$ day post CS was chosen based on the recommendation of specialists for the reasons that 1) PCSIs are most likely to happen within ten days and 2) if PCSI was suspected, the data collector could recommend patients to return to the hospital. The $30^{\text {th }}$ day post CS was chosen based on the recommendations from literature and CDC guidelines (Eggimann \& Pittet, 2001; Owens \& Stoessel, 2008; Petherick et al., 2006).

If a patient didn't answer a telephone call, the investigator made at least two attempts per day for the next five days. During the call, self-reported information from patients on surgical site infection signs was collected.

At the end of the study period, information from both forms was matched and combined to determine the PCSI rates.

\subsubsection{Dissemination of Surveillance Results}

The surveillance results were reported to the hospital's infection and prevention units, the maternity unit, and the hospital senior management team to review and prioritize their PCSI efforts.

\subsection{Data Analysis}

The overall PCSI rates, sub-divided by hospitalization and post-discharge periods, demographics, and types of PCSI, were summarized with descriptive statistics. PCSI rate was defined as: (the number of patients with PCSIs in 30 days)/(Number of patients admitted - lost to follow ups), as recommended by previous published literature (Noy \& Creedy, 2002).

\section{Results}

Between November 2017 and February 2018, 540 women underwent CS in the hospital and 536 (99.3\%) consented to participate in the surveillance. Among those who consented, 22 had no telephone and 174 could not be reached despite multiple attempts on the telephone. At the end of this study, a total 340 women completed the entire 30-day surveillance period (Table 1).

Table 1. Sample completed the surveillance

\begin{tabular}{ll}
\hline Patients & Number \\
\hline Number of CS surgery & 540 \\
\hline Number of women consented & 536 \\
\hline Lost to follow up & 196 \\
\hline Completed the entire surveillance & 340 \\
\hline
\end{tabular}


Our surveillance system detected a total of 38 women who developed PCSI infections within 30 days post-operation, making the overall PCSI rate 11.2\% (38/340). Among the 38 PCIS, 8 were detected during the hospitalization period. Post-discharge surveillance detected an additional 30 PCSIs, with 13 PCSI detected at the $10^{\text {th }}$ and 17 at the $30^{\text {th }}$ day phone calls (Table 2).

Table 2. PCSIs detected during hospitalization and post discharge periods

\begin{tabular}{|c|c|c|c|c|}
\hline & & & & $\mathrm{n}(\%)$ \\
\hline \multicolumn{3}{|c|}{ Sample (less lost to follow up) } & & 340 \\
\hline \multirow[t]{4}{*}{ PCSI } & During hospitalization & & 8 & 8 \\
\hline & Post-discharge period & $10^{\text {th }}$ day surveillance & 13 & \multirow{2}{*}{30} \\
\hline & & $30^{\text {th }}$ day surveillance & 17 & \\
\hline & Total & & & $38(11.2 \%)$ \\
\hline
\end{tabular}

Among the 38 women who developed PCSI, most of them were between 26 and 30 years old; $48.1 \%$ of them were in the overweight classification of BMI; $23.7 \%$ of them had no previous delivery; $57.9 \%$ had no previous cesarean section; $10.5 \%$ were in the social economic class I of poors (not able to afford basic needs); $81.8 \%$ had normal active labor duration; $94.7 \%$ did not have any pre-existing conditions of illness; $83.8 \%$ had emergency CS; and $81.6 \%$ were normally healthy patients (class 1 according to the American Society of Anesthesiologists classification system) (Table 3).

Among the 38 PCSIs, 32 (84.2\%) were superficial infections and 94.6\% of the surgical wounds were clean with risk of infection less than $2 \%$ immediately after CS (Class I of surgical wound). None of the women who developed PCSI experienced hemorrhage during their operations, $67.6 \%$ of the operations lasted less than one hour, and $97.4 \%$ of the women experiencing PCSI were given prophylaxis antibiotics (Table 3).

Table 3. Table summarizing the characteristics of women with PCSI

\begin{tabular}{|c|c|c|}
\hline Variable & & $\mathrm{n}(\%)$ \\
\hline \multirow[t]{4}{*}{ Age $(n=38)$} & $<=25$ years & $9(23.7 \%)$ \\
\hline & 26 to 30 years & $15(39.5 \%)$ \\
\hline & 31 to 35 years & $6(15.8 \%)$ \\
\hline & More than 35 years & $8(21.1 \%)$ \\
\hline \multirow[t]{3}{*}{ BMI (n=27) } & Normal & $4(14.8 \%)$ \\
\hline & Overweight & $13(48.1 \%)$ \\
\hline & Obese & $10(37.0 \%)$ \\
\hline \multirow[t]{6}{*}{ Previous number of Delivery $(\mathrm{n}=38)$} & 0 & $9(23.7 \%)$ \\
\hline & 1 & $7(18.4 \%)$ \\
\hline & 2 & $11(28.9 \%)$ \\
\hline & 3 & $6(15.8 \%)$ \\
\hline & 4 & $1(2.6 \%)$ \\
\hline & $5+$ & $4(10.5 \%)$ \\
\hline \multirow[t]{5}{*}{ Previous number of cesarean sections $(n=38)$} & 0 & $22(57.9 \%)$ \\
\hline & 1 & $11(28.9 \%)$ \\
\hline & 2 & $4(10.5 \%)$ \\
\hline & 3 & $1(2.6 \%)$ \\
\hline & $4+$ & $0(0 \%)$ \\
\hline
\end{tabular}




\begin{tabular}{|c|c|c|}
\hline \multirow[t]{3}{*}{ Socioeconomic category $(\mathrm{n}=38)$} & I & $4(10.5 \%)$ \\
\hline & II & $11(28.9 \%)$ \\
\hline & III & $23(60.5 \%)$ \\
\hline \multirow[t]{2}{*}{ Duration of Active labor $(n=33)$} & Normal $(<=12$ hours $)$ & $27(81.8 \%)$ \\
\hline & Prolonged (> 12 hours) & $6(18.2 \%)$ \\
\hline \multirow[t]{2}{*}{ Pre-existing condition $(\mathrm{n}=38)$} & Non & $36(94.7 \%)$ \\
\hline & Yes & $2(5.3 \%)$ \\
\hline \multirow[t]{2}{*}{ Type of operation $(n=37)$} & Elective & $6(16.2 \%)$ \\
\hline & Emergency & $31(83.8 \%)$ \\
\hline \multirow[t]{2}{*}{ Infection type $(\mathrm{n}=38)$} & Superficial & $32(84.2 \%)$ \\
\hline & Deep & $6(15.8 \%)$ \\
\hline \multirow[t]{3}{*}{ Rupture of membrane $(n=37)$} & No & $23(62.2 \%)$ \\
\hline & Yes, less than 24 hours & $12(32.4 \%)$ \\
\hline & Yes, greater than 24 hours & $2(5.4 \%)$ \\
\hline \multirow[t]{3}{*}{ ASA classification $(\mathrm{n}=38)$} & Class 1 & $31(81.6 \%)$ \\
\hline & Class 2 & $7(18.4 \%)$ \\
\hline & Class 3 & $0(0 \%)$ \\
\hline \multirow[t]{2}{*}{ Wound class $(\mathrm{n}=37)$} & Class I & $35(94.6 \%)$ \\
\hline & Class II & $2(5.4 \%)$ \\
\hline \multirow[t]{2}{*}{ Skin closure $(n=37)$} & Interrupted & $1(2.7 \%)$ \\
\hline & Not interrupted & $37(100 \%)$ \\
\hline Type of skin closure $(n=38)$ & Dissolvable suture & $38(100 \%)$ \\
\hline Hemorrhage $(\mathrm{n}=37)$ & No & $37(100 \%)$ \\
\hline \multirow[t]{2}{*}{ Duration of Operation $(n=37)$} & $<=1$ hour & $25(67.6 \%)$ \\
\hline & $>1$ hour & $12(32.4 \%)$ \\
\hline \multirow[t]{2}{*}{ Prophylaxis antibiotic given $(n=38)$} & No & $1(2.6 \%)$ \\
\hline & Yes & $37(97.4 \%)$ \\
\hline
\end{tabular}

\section{Discussion}

This project aimed to detect the true PCSI rate at a district hospital in Kigali. Since surgical site infection can develop within 30 days after an operative procedure, there was a need to establish a surveillance system to identify PCSI after patients were discharged from the hospital. Our surveillance system detected a total 38 women developing PCSI, making the PCSI rate $11.2 \%$. The PCSI rate established by the new system is consistent with the rates estimated in previous studies in Rwanda and surrounding country hospitals, which averaged from $4.9 \%$ to $10.9 \%$ (Bizimana et al., 2016; Chu, Maine, \& Trelles, 2015; Mpogoro et al., 2014).

Among the 38 PCSIs, only 8 (21\%) were developed during the hospitalization period; 30 (79\%) of the PCSI were detected after patients were discharged. This percentage is consistent with the findings from other studies, stating up to $95 \%$ of PCSI develop after women are discharged from hospitals (Owens \& Stoessel, 2008; Eggimann \& Pittet, 2001; Conroy et al., 2012; Bizimana et al., 2016; Mpogoro et al., 2014; Chu et al., 2015; Humphreys, 2009). Had our surveillance system followed the typical hospital surveillance methods restricted to recording only infections during the hospitalization period, we would have only detected 8 PCSI, making the PCSI rate $2.4 \%$ - substantially lower than the actual rate. If the hospital management were provided with this inaccurate data, their perceptions of the need for infection control measures might have been inaccurate.

The main advantage of our system was the ability to track PCSI developed after a patient was discharged from the hospital; this was especially important for this hospital, where the average length of hospital stay was 3 days for 
delivery cases. Although we failed to follow up on 196 patients, we were still able to track a relatively high number of infections - 30 PCSIs in the post-discharge period, which is more than 3 times than were recorded during the in-hospital period - illustrating the importance of having a system that can more accurately track PCSI. Since the system could only follow up with patients who were willing to participate after they were discharged, the actual PCSI rate is likely even higher than our system detected.

Another advantage of our system is its ease of use. We used the same CDC-defined criteria for identification of PCSIs, and the identification process did not require special diagnostic methods such as culture of fluid or tissue from the incision. The simple method we used, based on patient self-report, has been proved to be sensitive in detecting PCSI and is affordable for health facilities in LMIC (Bell \& Conway, 2015). Such simplicity is important particularly in resource-limited settings like our hospital, where laboratory supplies are not always available. We used clinical personnel of the hospital to track the PCSIs in order to minimize the extra training required to conduct the surveillance; their clinical knowledge enhanced their ability to identify infections. In addition, one of the data collectors we used was involved in the hospital infection prevention and control program and already has personal and professional interest in the surveillance; therefore, buy-in and support from the hospital and staff were high.

Such a surveillance system has great potential benefits for the hospital for both planning and operations. Studies have shown that post-discharge surgical site infections are associated with a high readmission rates (Gibson, Tevis, \& Kennedy, 2014); therefore, having more accurate PCSI information can prepare hospital management to appropriately allocate resources for prevention as well as for timely and early treatment of PCSI, especially since the cost associated with any type of surgical site infection is known to be high (Badia et al., 2017). Apart from the time and salary of data collectors, the only added cost of the surveillance system was the fee for telephone calls.

Another added value of the system is that contacting patients for follow up after discharge establishes a relationship and trust between the hospital and the community. During the telephone calls, many patients expressed their astonishment that the hospital was taking care of them even after discharge.

Comprehensive PCSI surveillance that fully covered the 30-day post-surgical period was effective in estimating a more accurate PCSI rate; however, our study did face substantial loss to follow up. This likely affected the observed PCSI rate. Furthermore, since the data collectors were collecting PCSI information in addition to performing their regular hospital duties, they could only make phone calls when they had free time or after their work shifts. This could have contributed to the high-loss to follow up. Allocating staff to take on this responsibility may improve the response rate. Patients could also be asked to provide their preferred time for telephone calls.

\section{Conclusion}

PCSI remains a burden in LMICs, and it is important for hospitals to have accurate PCSI information in order to develop better infection control and response plans. The PCSI surveillance system developed in this project covered the 30-day period after surgery, thus providing a more accurate estimate of PCSI rate. The cost to the hospital was relatively small but the system could be labor intensive. Long term sustainability of the project must be evaluated. Other hospitals in LMICs could adopt the process to establish an accurate PCSI surveillance system in order to make informed decisions about allocating human and financial resources to reduce PCSI.

\section{Acknowledgement}

We acknowledge the contribution of nurses at Kacyiru hospital who helped with data collection on mothers who delivered through C-section at that hospital. Our gratitude is addressed to the lecturers at the University of Global Health Equity (UGHE) and our colleague (Joy Atwine) at Management Sciences for Health (MSH) for valuable advice on the study. We are also grateful to Nadia Hitimana, our colleague at MSH, who helped with proof reading of the study report.

\section{References}

Allegranzi, B., \& Pittet, D. (2008). Preventing infections acquired during health-care delivery. The Lancet, 372(9651), 1719-1720. https://doi.org/10.1016/S0140-6736(08)61715-8

Badia, J. M., Casey, A. L., Petrosillo, N., Hudson, P. M., Mitchell, S. A., \& Crosby, C. (2017). Impact of surgical site infection on healthcare costs and patient outcomes: a systematic review in six European countries. Journal of Hospital Infection, 96(1), 1-15. https://doi.org/10.1016/j.jhin.2017.03.004

Bagnall, N. M., Vig, S., \& Trivedi, P. (2009). Surgical-site infection. Surgery. https://doi.org/10.1016/j.mpsur.2009.08.007

Bell, B. P., \& Conway, P. (2015). Adherence to the CDC's Infection Definitions and Criteria is Needed to Ensure 
Accuracy, Completeness, and Comparability of Infection Information (Cdc), pp. 1-3. Retrieved from http://www.cdc.gov/nhsn/pdfs/cms/nhsn-reporting-signed.pdf

Bizimana, K., Ndoli, J., Bayingana, C., Baluhe, I., Gilson, G. J., \& Habimana, E. (2016). Prevalence and Risk Factors for Post Cesarean Delivery Surgical Site Infection in a Teaching Hospital Setting in Rural Rwanda: A Prospective Cross Sectional Study. International Journal of Current Microbiology and Applied Sciences, 5(6), 631-641. https://doi.org/10.20546/ijcmas.2016.506.069

Chu, K., Maine, R., \& Trelles, M. (2015). Cesarean section surgical site infections in sub-Saharan Africa: A multi-country study from Medecins sans Frontieres. World Journal of Surgery, 39(2), 350-355. https://doi.org/10.1007/s00268-014-2840-4

Conroy, K., Koenig, A. F., Yu, Y.-H., Courtney, A., Lee, H. J., \& Norwitz, E. R. (2012). Infectious morbidity after cesarean delivery: 10 strategies to reduce risk. Reviews in Obstetrics \& Gynecology, 5(2), 69-77.

Dhar, H., Al-Busaidi, I., Rathi, B., Nimre, E. A., Sachdeva, V., \& Hamdi, I. (2014). A study of post-caesarean section wound infections in a regional referral hospital, Oman. Sultan Qaboos University Medical Journal, 14(2), 211-217.

Eggimann, P., \& Pittet, D. (2001). Infection control in the ICU. Chest, 120(6), 2059-2093. https://doi.org/10.1378/chest.120.6.2059

Gibson, A., Tevis, S., \& Kennedy, G. (2014). Readmission after delayed diagnosis of surgical site infection: A focus on prevention using the American College of Surgeons National Surgical Quality Improvement Program. American Journal of Surgery, 207(6), 832-839. https://doi.org/10.1016/j.amjsurg.2013.05.017

Gould, D. (2007). Caesarean section, surgical site infection and wound management. Nursing Standard (Royal College of Nursing (Great Britain): 1987), 21(32), 57-8. https://doi.org/10.7748/ns2007.04.21.32.57.c4498

Gur, R., Duggal, S. D., Rongpharpi, S. R., Srivastava, R., Kumar, A., Gupta, V., ... Pundhir, S. (2015). Post caesarean surgical site infections. Archives of Clinical Microbiology, 6(1), 1-6. https://doi.org/10.1177/0192513X07300968

Haley, R. W., Culver, D. H., White, J. W., Morgan, W. M., Emori, T. G., Munn, V. P., \& Hooton, T. M. (1985). The Efficacy of Infection Surveillance and Control Programs in Preventing Nosocomial Infections in Us Hospitals. Am. J. Epidemiol., 121(2), 182-205. https://doi.org/10.1093/oxfordjournals.aje.a113990

Humphreys, H. (2009). Preventing surgical site infection. Where now?. Journal of Hospital Infection, 73(4), 316-322. https://doi.org/10.1016/j.jhin.2009.03.028

Kelly, K. N., \& Monson, J. R. T. (2012). Hospital-acquired infections. Surgery (Oxford), 30(12), 640-644. https://doi.org/10.1016/j.mpsur.2012.10.005

Lukas, S., Hogan, U., Muhirwa, V., Davis, C., Nyiligira, J., Ogbuagu, O., \& Wong, R. (2016). Establishment of a hospital-acquired infection surveillance system in a teaching hospital in Rwanda. International Journal of Infection Control, 12(3), 1-9. https://doi.org/10.3396/ijic.v12i3.018.16

Maruta, A. (2015, December). Surveillance of Surgical Site Infections following Caesarean Section at Two Central Hospitals in Harare, Zimbabwe, pp. 6-105. Retrieved from https://scholar.sun.ac.za

Mitt, P., Lang, K., Peri, A., Maimets, M., \& National, P. (2002). S Urgical -S Ite I Nfections F Ollowing C Esarean S Ection in an E Stonian U Niversity H Ospital: P Ostdischarge S Urveillance and. Infection Control and Hospital Epidemiology, 26(5), 449-454. https://doi.org/10.1086/502566

Mpogoro, F. J., Mshana, S. E., Mirambo, M. M., Kidenya, B. R., Gumodoka, B., \& Imirzalioglu, C. (2014). Incidence and predictors of surgical site infections following caesarean sections at Bugando Medical Centre, Mwanza, Tanzania. Antimicrobial Resistance and Infection Control, 3(1). https://doi.org/10.1186/2047-2994-3-25

Nguhuni, B., De Nardo, P., Gentilotti, E., Chaula, Z., Damian, C., Mencarini, P., ... Ippolito, G. (2017). Reliability and validity of using telephone calls for post-discharge surveillance of surgical site infection following caesarean section at a tertiary hospital in Tanzania. Antimicrobial Resistance \& Infection Control, 6(1), 43. https://doi.org/10.1186/s13756-017-0205-0

Noy, D., \& Creedy, D. (2002). Postdischarge surveillance of surgical site infections: A multi-method approach to data collection. American Journal of Infection Control, 30(7), 417-424. 
https://doi.org/10.1067/mic.2002.123393

Owens, C. D., \& Stoessel, K. (2008). Surgical site infections: epidemiology, microbiology and prevention. Journal of Hospital Infection, 70(SUPPL. 2), 3-10. https://doi.org/10.1016/S0195-6701(08)60017-1

Petherick, E. S., Dalton, J. E., Moore, P. J., \& Cullum, N. (2006). Methods for identifying surgical wound infection after discharge from hospital: a systematic review. BMC Infectious Diseases, 6(1), 170. https://doi.org/10.1186/1471-2334-6-170

Saskatchewan Ministry of Health. (2015). Surgical Site Infection (SSI) Surveillance Protocol: Saskatchewan. Saskatchewan. Retrieved from https://www.ehealthsask.ca/services/resources/Resources/SSI Surveillance Protocol - April 2015 (1).pdf

Soto-Vega, E., Casco, S., K, C., Flores-Hernández, D., Landini, V., \& Guillén-Florez, A. (2015). Rising Trends of Cesarean Section Worldwide: A Systematic Review. Obstet Gynecol International Journal, 3(2). https://doi.org/10.15406/ogij.2015.03.00073

Srun, S., Sinath, Y., Seng, A. T., Chea, M., Borin, M., Nhem, S., ... Goyet, S. (2013). Surveillance of post-caesarean surgical site infections in a hospital with limited resources, Cambodia. Journal of Infection in Developing Countries, 7(8), 579-585. https://doi.org/10.3855/jidc.2981

Taylor, E. W., Duffy, K., Lee, K., Noone, A., Leanord, A., King, P. M., \& O’Dwyer, P. (2003). Telephone call contact for post-discharge surveillance of surgical site infections. A pilot, methodological study. Journal of Hospital Infection, 55(1), 8-13. https://doi.org/10.1016/S0195-6701(03)00217-2

Yadav, S., Kaur, S., Yadav, S. S., \& Thakur, B. (2016). Analysis of caesarean rate, indications and complications: review from medical college Ambala, Haryana, India. Int J Reprod Contracept Obstet Gynecol, 5(10), 3326-3329.

Yaya, S., Uthman, O. A., Amouzou, A., \& Bishwajit, G. (2018). Disparities in caesarean section prevalence and determinants across sub-Saharan Africa countries. Global Health Research and Policy, 3(19), 1-9. https://doi.org/10.1186/s41256-018-0074-y

\section{Appendix 1. In-Patient and Readmissions Post Cesarean Section SSIs Surveillance - Case Report Form}

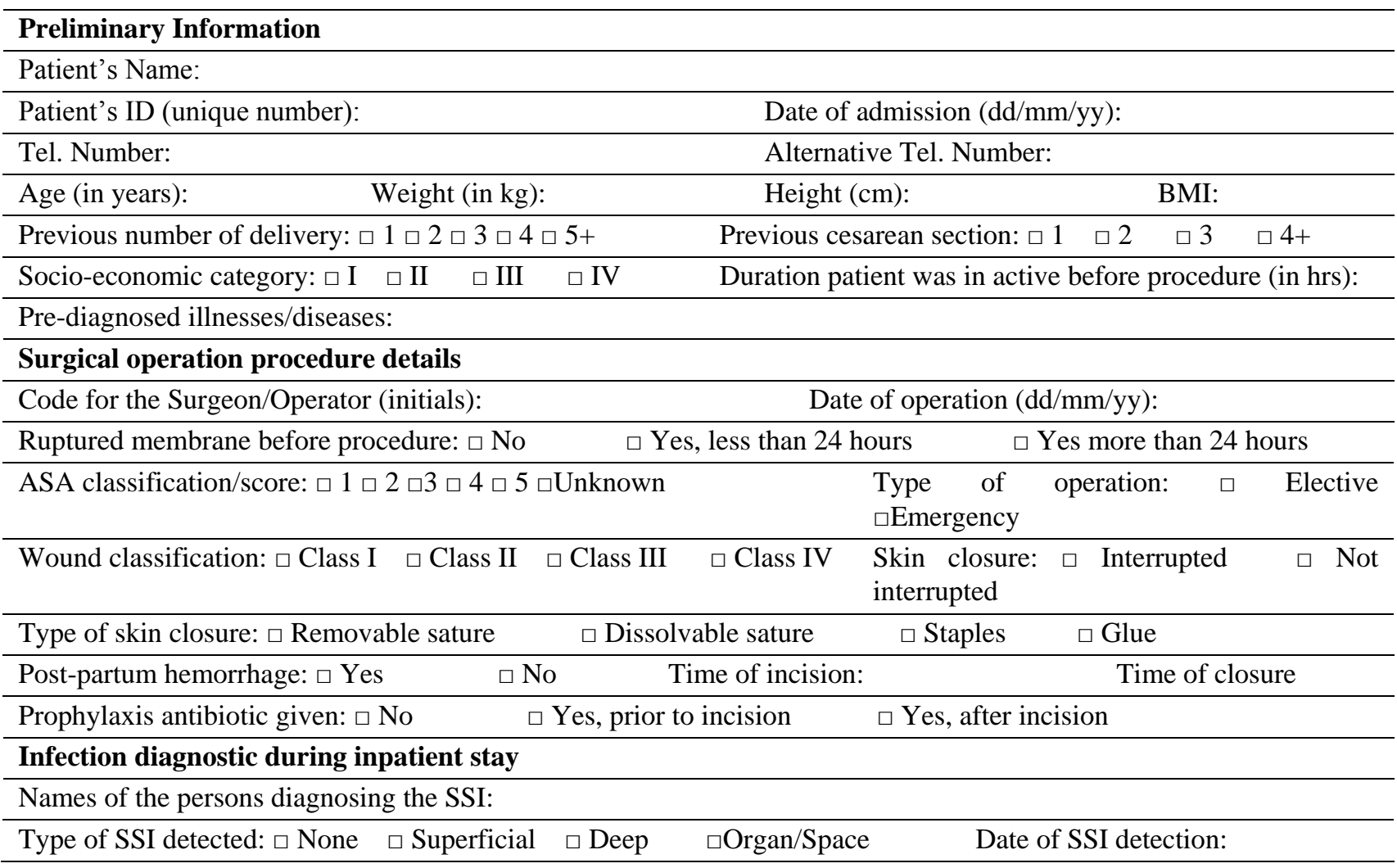




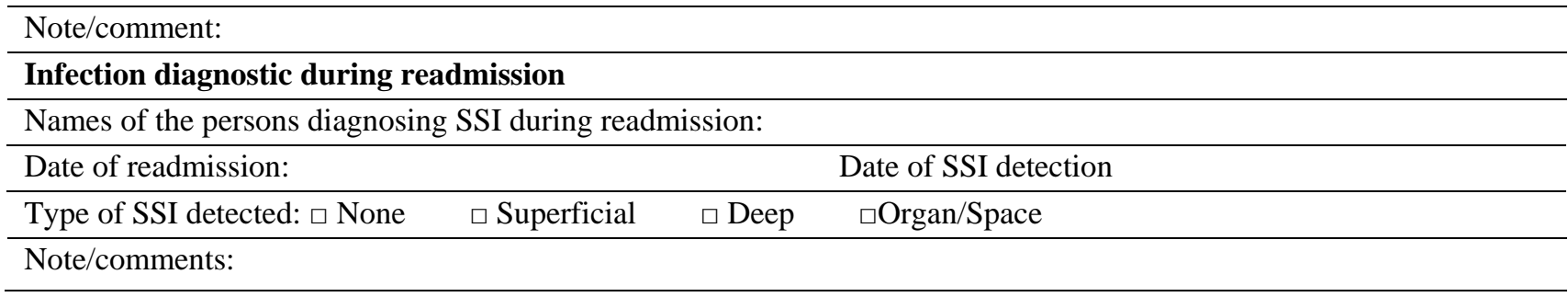

\section{Appendix 2. Post Discharge Telephone Surveillance of Post Cesarean SSIs}

\section{$\square 10^{\text {th }} \quad \square 30^{\text {th }}$ day after surgical operation}

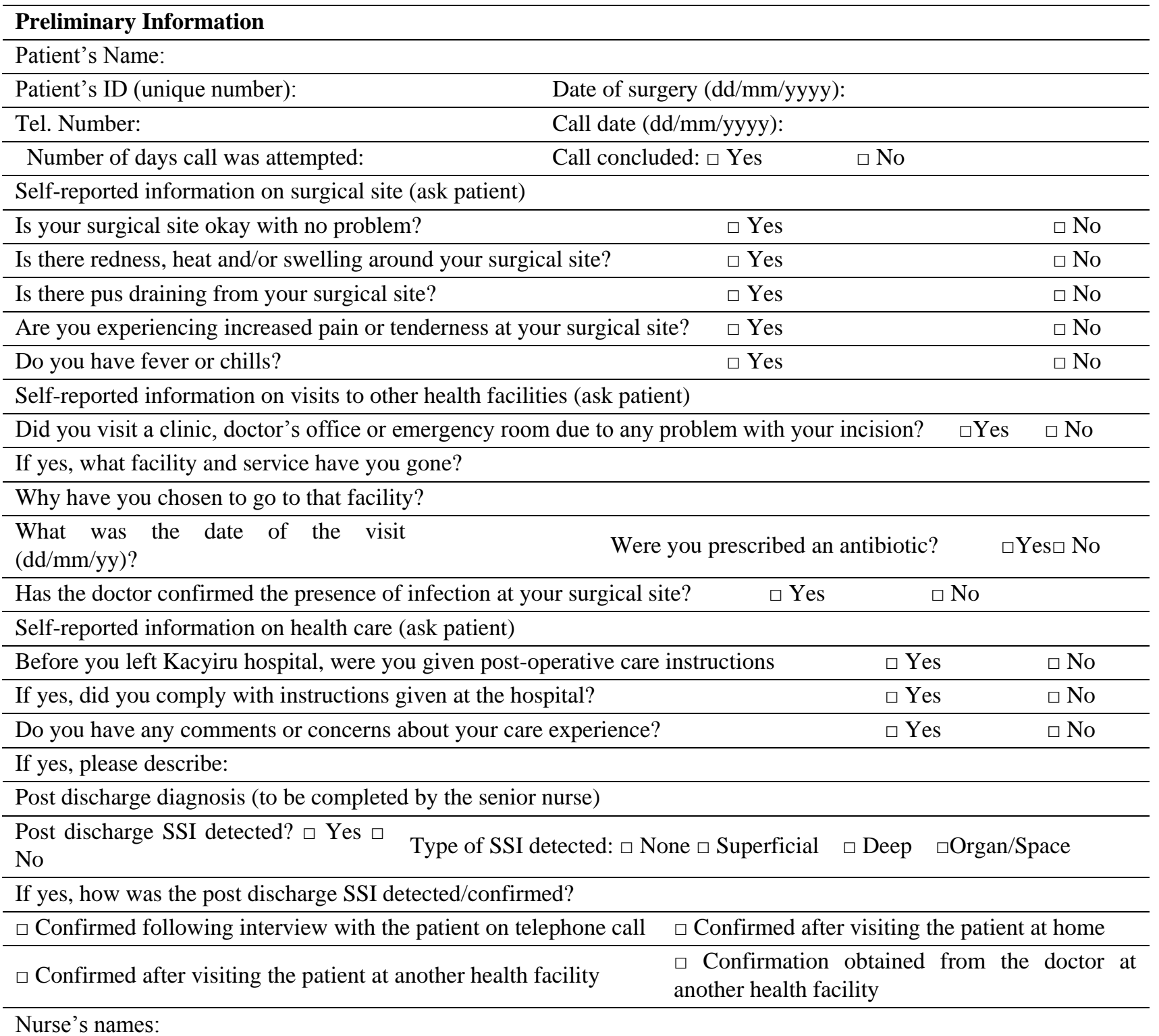

Note/Comments: 